\title{
Fruquintinib in Combination with PD-1 Inhibitors in Patients with Refractory Non-MSI-H/pMMR Metastatic Colorectal Cancer: A Real-World Study in China
}

miaomiao GOU ( $\square$ goumiaomiaode@163.com )

Chinese PLA General Hospital

Niansong Qian

Chinese PLA General Hospital

Yong Zhang

Chinese PLA General Hospital

Huan Yan

Chinese PLA General Hospital

Haiyan Si

Chinese PLA General Hospital

zhikuan wang

Chinese PLA General Hospital

Guanghai Dai

Chinese PLA General Hospital

\section{Research Article}

Keywords:

Posted Date: February 17th, 2022

DOI: https://doi.org/10.21203/rs.3.rs-1113734/v1

License: (c) (i) This work is licensed under a Creative Commons Attribution 4.0 International License. Read Full License 


\section{Abstract}

Background: Fruquintinib, a vascular endothelial growth factor receptor inhibitor, is a new anti-cancer drug independently developed in China to treat refractory metastatic colorectal cancer (mCRC). In Japan, regorafenib in combination with nivolumab has demonstrated promise in patients with refractory $\mathrm{mCRC}$. Here, in a real-world study, we aimed to evaluate the efficacy of fruquintinib plus various programmed death-1 (PD-1) inhibitors after standard treatment in Chinese non- microsatellite instability-high(MSIH)/mismatch repair proficient mCRC patients.

Methods: Thirty-five patients with refractory mCRC were involved in the study. They were given fruquintinib ( 3 or $5 \mathrm{mg}$ orally, once daily for 3 weeks followed by 1 week off in 4 week cycles) and a PD-1 inhibitor. Progression free survival (PFS), overall survival (OS), disease control rate (DCR), and objective response rate (ORR) were reviewed and evaluated.

Results: Of the 35 patients, the median age was 54 years (29-85). The ORR was $11.4 \%$ (4/35), DCR was $60 \%(21 / 35)$, median PFS was 3.8 months, and median OS was 14.6 months. The duration of response was 3.4 months. The PFS between left and right primary tumors as well as whether lung metastases were present did not differ $(p>0.05)$, which is inconsistent with the REGONIVO study. The multivariate analysis indicated no association of OS benefit in the specified subgroups. No adverse-effect-related deaths were reported.

Conclusions: Fruquintinib in combination with anti-PD-1 was observed to have clinical activity in a small population of patients with heavily pretreated mCRC. The improvements in OS need to be verified using more real-world medical data.

\section{Introduction}

Colorectal cancer (CRC) is the fourth most common type of cancer and fifth leading cause of cancerrelated deaths in China ${ }^{[1]}$. Despite improvements in treatments for advanced CRC, the survival of these patients following one or two previous standard lines of therapy remains dismal. Currently, the United States Food and Drug Administration has approved TAS-102 and regorafenib as third line therapies for advanced CRC patients ${ }^{[2,3]}$. However, the CONCUR ${ }^{[4]}$ and TERRA studies ${ }^{[5]}$ reported an average progression-free survival (PFS) of three months in Asians. Consequently, attempts were made to improve the survival benefit. In 2019, REGONIVO study ${ }^{[6]}$ demonstrated that nivolumab plus regorafenib led to encouraging activity and manageable safety for patients with late-stage microsatellite stability (MSS) or mismatch repair proficient (pMMR) gastric cancer and CRC. Regorafenib ${ }^{[7]}$ is a novel oral multi-kinase inhibitor that blocks the activity of several protein kinases, including those involved in the regulation of tumor angiogenesis (vascular endothelial growth factor receptor [VEGFR]1, VEGFR2, VEGFR3, and TIE2) and oncogenesis (KIT, RET, RAF1, BRAF, and BRAFV600E). Nivolumab is a human immunoglobulin G(4)blocking antibody that inhibits the T-cell programmed death-1 (PD-1) checkpoint protein. It has demonstrated encouraging function of tumor control in several cancers whether given alone or in 
combination with other agents $^{[8-11]}$. The combination of a PD-1 inhibitor with angiogenesis agents seems to aid in tumor control, as suggested from the REGONIVO study.

Fruquintinib, an oral multi-kinase inhibitor, is a potent, highly selective small-molecule inhibitor of VEGFR1, VEGFR2, and VEGFR3. Based on the FRESCO study and the 2019 Chinese Society of Clinical Oncology guidelines, it is recommended as a treatment among Chinese metastatic CRC (mCRC) patients who experienced tumor progression following two or more prior chemotherapy regimens ${ }^{[12,13]}$. Currently, at least six types of PD-1 inhibitors are available clinically. Data on the efficacy of fruquintinib in combination with anti-PD-1 inhibitors for MSS/pMMR mCRC in a real-world setting have yet to be reported. Here, we sought to further explore, retrospectively in our clinic center, the efficacy profiles of fruquintinib plus PD-1 inhibitors as well as the potential clinical correlates of benefits in patients with metastatic MSS/pMMR CRC that had progressed following second-line or subsequent treatment.

\section{Patients And Methods}

Patients in the oncology department of the Chinese PLA General Hospital from January 2019 to August 2021 who progressed to standard chemotherapy were enrolled. The eligibility criteria were: patients with histological or cytological confirmation of adenocarcinoma of the colon or rectum; they had experienced failure of first and second standard therapies such as FOLFOX (fluoropyrimidine and oxaliplatin) or FORFIRI (irinotecan and fluoropyrimidine) with or without bevacizumab or cetuximab; they had at least one non-resectable measurable lesion to evaluate treatment response; and they were administered at least two cycles of treatment. The exclusion criteria included: patients with less than one cycle of treatment, and those with little information on tumor response. This retrospective study was approved by the independent ethics committee of our hospital((NO:S2019-201-01) .

Fruquintinib was given orally once daily in 28-day (D) cycles (21D on/7D off). The PD-1 inhibitor (200 mg pembrolizumab, $3 \mathrm{mg} / \mathrm{kg}$ nivolumab, $200 \mathrm{mg}$ sintilimab or camrelizumab, or $240 \mathrm{mg}$ toripalimab was given intravenously once every 3 weeks) on D1. The fruquintinib starting dose was $5 \mathrm{mg}$; if it was not well tolerated, it was deescalated to 3 or $4 \mathrm{mg}$ in the therapy cycle.

\section{Assessments}

Patients were followed up until the cutoff date of August 1, 2021. Tumor evaluation was based on RECIST (version 1.1). The response evaluation included complete response (CR), partial response (PR), stable disease (SD), and progression disease (PD). The objective response rate (ORR) and disease control rate $(D C R)$ were also evaluated. The ORR was calculated as the $C R+P R$, while the $D C R$ was the $C R+P R$ + SD.

The primary endpoint was PFS, which was defined as the time from treatment to the RECIST-defined disease progression. The second endpoint was the duration of response (DOR), the time from the first 
response to disease progression or death from any cause, whichever occurred first. The overall survival (OS) was the time from third line treatment to death from any cause.

Exploratory univariate analyses were performed using the log-rank test, which compared different clinical variables such as tumor location (left versus right side), KRAS status (wild or mutation), liver and lung metastases, and other variables.

\section{Statistical Analysis}

SPSS software 18.0 was used for statistical analysis. Objective response and disease control were presented as proportions and comparisons between groups were carried out using the stratified k-square test. Kaplan-Meier curves were used to estimate median OS, PFS, and DOR. Differences between clinic features in PFS and OS were assessed using the log-rank test. The statistical significance level was set to 0.05 , and confidence intervals (Cls) were set to $95 \%$.

\section{Results}

From January 2019 to August 2021, 35 patients were enrolled in this retrospective study at our hospital's oncology department. Patient clinical characteristics are listed in Table 1. At baseline, the average age was 54 years old (range 29-85), and $77.1 \%$ had ECOG performance status (PS) scores of $0 / 1.80 \%$ of the patients had liver metastases, while $42.9 \%$ had lung metastases. The primary tumor site was in the left colon in $77.1 \%$ of cases and the right in $22.9 \% .88 .6 \%$ of patients had received bevacizumab prior to treatment. Finally, 23 patients had KRAS mutations, and all patients achieved MSS.

The median number of cycles was 5.0 (range 2-24); four patients had to deescalate fruquintinib from 5 $\mathrm{mg}$ to $3 \mathrm{mg}$. Six patients started fruquintinib at $3 \mathrm{mg}$; one discontinued the treatment because of an adverse event. $62.8 \%$ (22/35) patients received sintilimab plus fruquintinib. Five patients were given pembrolizumab, two patients with nivolumab, and four with camrelizumab.

In terms of response, no patient had a CR. Four patients achieved PRs, and 17 patients had SD. Fourteen achieved PD in accordance with RECIST version 1.1. DCR was 60\%, and ORR was $11.4 \%$ (Table 2, Fig. 1). The median PFS was 3.8 months ( $95 \%$ Cl: $1.8-5.8$ months), and the OS was 14.6 months ( $95 \% \mathrm{Cl}: 5.4-$ 23.8 months; Table 2, Figs. 2-3). The median DOR was 3.4 months (Table 2, Fig. 4).

The univariate analysis indicated that the PFS between left versus right primary tumors as well as whether lung metastases were present was not significantly different $(p=0.396$ and $p=0.562$, respectively). Neither KRAS mutation nor liver metastases were associated with better PFS or OS (Tables 3-4). The same trends were demonstrated using multivariate analysis.

\section{Discussion}


Patients with mCRC will eventually face challenges in its medical management following failure of standard treatment. However, limited choices with good performance still exist in third or further line therapy. Previous studies have found that MCRC patients harboring MSS show little response to immunotherapy ${ }^{[14]}$, likely due to a complex tumor microenvironment that counteracts antitumor immunity via a combination of low antigenic tumor cells and an immunosuppressive tumor microenvironment ${ }^{[15]}$. However, the REGONIVO study demonstrated promising anticancer activity in mCRC patients with MSS who were administered with a PD-1 inhibitor (nivolumab) and regorafenib; these findings have a profound impact on cancer care for mCRC patients. As of January 2020, three additional antibody therapeutics developed by Chinese companies (tislelizumab, sintilimab, and camrelizumab) became available in China ${ }^{[16]}$. These compounds are highly selective, fully humanized monoclonal antibodies that block the interaction between PD-1 and its ligands ${ }^{[17]}$. Based on the REGONIVO combination strategy, our center attempted a similar regime using fruquintinib in combination with various PD- 1 inhibitors as a third line therapy for MCRC patients with MSS. We retrospectively reviewed those patients and analyzed the efficacy of their treatments.

We obtained medical records from 35 patients at the time of analysis. Broadly, our therapy regime had efficacy. DCR was $60 \%$, and ORR was $11.4 \%$ with four PRs observed. In the REGONIVO study, ORR was observed in $40 \%$ of patients; among 50 patients, including $25 \mathrm{CRC}$ and 25 gastric cancers, eight patients had PRs, resulting in an ORR of $33 \%$ in MSS CRC patients. By contrast, our response rates were nonsuperior to the REGONIVO study. Notably, in the North American population, the efficacy of the combination treatment also differed from the Japanese population. More specifically, in the North American version ${ }^{[18]}$ of REGONIVO, five patients (7.1\%) out of 70 had a PR, and $22(31.4 \%)$ had SD. The LEAP-005 study ${ }^{[19]}$ (NCT03797326) evaluated the efficacy and safety of lenvatinib plus pembrolizumab in patients with previously treated CRC. They found an ORR of $22 \%(95 \% \mathrm{Cl}: 9-40)$ and a DCR of $47 \%$ in 32 CRC patients. Such discrepancies may be due to the REGONIVO study being a dose-finding and doseexpansion phase $1 \mathrm{~b}$ trial with an aim of exploring safety and recommended doses. Accordingly, response rates should be verified in further investigations using a larger cohort. Second, the efficacy of the combination treatment varies depending on the specific anti-PD-1 or angiogenesis agent and the population. Third, patients in trials normally have PS scores of $0-1$. Real-world studies do not limit PS scores, which means that our study included patients with worse PS scores, which may have affected the treatment responses.

We obtained a median PFS of 3.8 months. This result seems disappointing. The PFS was not superior to that from the REGONIVO study, and much similar to other studies associated with third or subsequent line treatment for mCRC. The PFS in the REGONIVO study was 6.3 months ${ }^{[6]}, 3.7$ months in the FRESCO ${ }^{[20]}$ study, and 2.0 months in the TAS-102 study ${ }^{[5]}$. We acknowledge the non-superior PFS of fruquintinib and anti-PD-1 treatment in such comparisons. Real world studies are complex, with various factors that reflect actual clinical practice, whereas clinical trials exclude poor condition. Furthermore, although regorafenib and fruquintinib are the same type of oral anti-angiogenesis agents, their underlying mechanisms at the functional site are different. Regorafenib is multi-targeted ${ }^{[7]}$, while fruquintinib is highly selective for 
VEGFR1, VEGFR2, and VEGFR3 ${ }^{[12]}$. The molecular properties of PD-1-targeted antibodies are another factor. We acknowledge the limitations in comparing nivolumab and other types of PD-1 antibodies. However, nivolumab and pembrolizumab differ in the extent and spatial location of their binding sites with the flexible PD-1 loops ${ }^{[21]}$. Based on the available characterization data on anti-PD-1 antibodies, the molecular behavior between nivolumab and other anti-PD-1 antibodies likely differ ${ }^{[22,23]}$. We also consider, to some extent, the effect of fruquintinib dose on efficacy. Some patients in our study took fruquintinib at $3 \mathrm{mg}$ due to their tolerance, while the recommended dose on a continuous regimen is $5 \mathrm{mg}$ QD ${ }^{[24,25]}$. Therefore, the dose may have affected clinical efficacy.

Notably, the median OS in our study was much higher than in previously reported studies. The OS benefit in our real-world study was 14.6 months, while that of the FRESCO study was 9.3 months, which was the longest OS reported prior to our study. We cannot directly compare the efficacy of our study with the FRESCO or REGONIVO studies; this outcome needs to be confirmed in a larger group.

The PFS in our study appear to be no better than the results from the FRESCO study. Accordingly, we further assessed whether clinical characteristics were correlated with clinical outcome. Gender, tumor location, metastatic organs, and KRAS status did not significantly differ, which was inconsistent with the REGONIVO study. All patients responding in the REGONIVO study ${ }^{[6]}$ were male with lung metastases and had PS scores of 0 . Therefore, the results from the REGONIVO study may have been biased by the small sample and need further confirmation in a larger population.

One shortcoming of our study is the limited population and the absence of a side effects profile and data on PD-L1 expression. We did not have sufficient data to calculate a safety profile; what we know is that we found no severe adverse-related deaths. PD-L1 is believed to indicate response to anti-PD-1 antibodies in several tumors ${ }^{[26]}$, However, data are lacking in PD-L1 expression; thus, we were unable to assess PDL1 as a potential biomarker for CRC patients.

In conclusion, we found that fruquintinib in combination with anti-PD-1 had clinical activity in mCRC refractory to standard chemotherapy. However, this benefit was not observed across all prespecified patient subgroups. Further research is needed to assess OS benefits using a larger group.

\section{Declarations}

Ethics approval and consent to participate: This retrospective study was approved by the independent ethics committee of Chinese PLA General Hospital and all the authors consent to participate to the study.

Consent for publication: all the authors had consent for publication.

Availability of data and material: data and material are availability when required

Competing interests: we declare no conflicts of interest. 
Funding: There was no funding.

Authors' contributions: Miaomiao Gou, Niansong Qian, Yong Zhang are in charge of data collection and writing, Huan Yan and Haiyan Si contribute to data collection, Zhikuan Wang and Guanghai Dai come up thoughts.

Acknowledgements: Thanks for all the works of authors.

\section{References}

1. Siegel RL, Miller KD, Jemal A. Cancer statistics, 2020. CA Cancer J Clin. 2020. 70(1): 7-30.

2. Mayer RJ, Van Cutsem E, Falcone A, et al. Randomized trial of TAS-102 for refractory metastatic colorectal cancer. N Engl J Med. 2015. 372(20): 1909-19.

3. Grothey A, Van Cutsem E, Sobrero A, et al. Regorafenib monotherapy for previously treated metastatic colorectal cancer (CORRECT): an international, multicentre, randomised, placebocontrolled, phase 3 trial. Lancet. 2013. 381(9863): 303-12.

4. Li J, Qin S, Xu R, et al. Regorafenib plus best supportive care versus placebo plus best supportive care in Asian patients with previously treated metastatic colorectal cancer (CONCUR): a randomised, double-blind, placebo-controlled, phase 3 trial. Lancet Oncol. 2015. 16(6): 619-29.

5. Xu J, Kim TW, Shen L, et al. Results of a Randomized, Double-Blind, Placebo-Controlled, Phase III Trial of Trifluridine/Tipiracil (TAS-102) Monotherapy in Asian Patients With Previously Treated Metastatic Colorectal Cancer: The TERRA Study. J Clin Oncol. 2018. 36(4): 350-358.

6. Fukuoka S, Hara H, Takahashi N, et al. Regorafenib Plus Nivolumab in Patients With Advanced Gastric or Colorectal Cancer: An Open-Label, Dose-Escalation, and Dose-Expansion Phase lb Trial (REGONIVO, EPOC1603). J Clin Oncol. 2020. 38(18): 2053-2061.

7. Ettrich TJ, Seufferlein T. Regorafenib. Recent Results Cancer Res. 2018. 211: 45-56.

8. Borghaei H, Paz-Ares L, Horn L, et al. Nivolumab versus Docetaxel in Advanced Nonsquamous NonSmall-Cell Lung Cancer. N Engl J Med. 2015. 373(17): 1627-39.

9. El-Khoueiry AB, Sangro B, Yau T, et al. Nivolumab in patients with advanced hepatocellular carcinoma (CheckMate 040): an open-label, non-comparative, phase 1/2 dose escalation and expansion trial. Lancet. 2017. 389(10088): 2492-2502.

10. Rizvi NA, Mazières J, Planchard $D$, et al. Activity and safety of nivolumab, an anti-PD-1 immune checkpoint inhibitor, for patients with advanced, refractory squamous non-small-cell lung cancer (CheckMate 063): a phase 2, single-arm trial. Lancet Oncol. 2015. 16(3): 257-65.

11. Hodi FS, Chiarion-Sileni V, Gonzalez R, et al. Nivolumab plus ipilimumab or nivolumab alone versus ipilimumab alone in advanced melanoma (CheckMate 067): 4-year outcomes of a multicentre, randomised, phase 3 trial. Lancet Oncol. 2018. 19(11): 1480-1492.

12. Shirley M. Fruquintinib: First Global Approval. Drugs. 2018. 78(16): 1757-1761. 
13. CSOCOC DATGFCCWG. Chinese Society of Clinical Oncology (CSCO) diagnosis and treatment guidelines for colorectal cancer 2018 (English version). Chin J Cancer Res. 2019. 31(1): 117-134.

14. Le DT, Uram JN, Wang H, et al. PD-1 Blockade in Tumors with Mismatch-Repair Deficiency. N Engl J Med. 2015. 372(26): 2509-20.

15. Kather JN, Halama N, Jaeger D. Genomics and emerging biomarkers for immunotherapy of colorectal cancer. Semin Cancer Biol. 2018. 52(Pt 2): 189-197.

16. Kaplon H, Reichert JM. Antibodies to watch in 2019. MAbs. 2019. 11(2): 219-238.

17. Hoy SM. Sintilimab: First Global Approval. Drugs. 2019. 79(3): 341-346.

18. Marwan Fakih KPSR, David Z. Chang JCB, Timothy Larson ALC, et al. Single-arm, phase 2 study of regorafenib plus nivolumab in patients with mismatch repair-proficient (pMMR)/microsatellite stable (MSS) colorectal cancer (CRC).

19. Carlos A. Gomez-Roca EY, Seock-Ah Im ECA, Hélène Senellart MD, et al. LEAP-005: A phase 2 multicohort study of lenvatinib plus pembrolizumab in patients with previously treated selected solid tumors-Results from the colorectal cancer cohort. Journal of Clinical Oncology 2021 39:15_suppl, 3564-3564..

20. Li J, Qin S, Xu RH, et al. Effect of Fruquintinib vs Placebo on Overall Survival in Patients With Previously Treated Metastatic Colorectal Cancer: The FRESCO Randomized Clinical Trial. JAMA. 2018. 319(24): 2486-2496.

21. Fessas P, Lee H, Ikemizu S, Janowitz T. A molecular and preclinical comparison of the PD-1-targeted T-cell checkpoint inhibitors nivolumab and pembrolizumab. Semin Oncol. 2017. 44(2): 136-140.

22. Brahmer JR, Drake CG, Wollner I, et al. Phase I study of single-agent anti-programmed death-1 (MDX1106) in refractory solid tumors: safety, clinical activity, pharmacodynamics, and immunologic correlates. J Clin Oncol. 2010. 28(19): 3167-75.

23. Davies AM, Rispens T, Ooijevaar-de HP, et al. Structural determinants of unique properties of human IgG4-Fc. J Mol Biol. 2014. 426(3): 630-44.

24. Cao J, Zhang J, Peng W, et al. A Phase I study of safety and pharmacokinetics of fruquintinib, a novel selective inhibitor of vascular endothelial growth factor receptor-1, -2 , and -3 tyrosine kinases in Chinese patients with advanced solid tumors. Cancer Chemother Pharmacol. 2016. 78(2): 259-69.

25. Xu RH, Li J, Bai Y, et al. Safety and efficacy of fruquintinib in patients with previously treated metastatic colorectal cancer: a phase $\mathrm{lb}$ study and a randomized double-blind phase II study. J Hematol Oncol. 2017. 10(1): 22.

26. Patel SP, Kurzrock R. PD-L1 Expression as a Predictive Biomarker in Cancer Immunotherapy. Mol Cancer Ther. 2015. 14(4): 847-56.

\section{Tables}

Table 1 Baseline Characteristics of patients $(n=35)$ 
Characteristics

Total

Gender

\begin{tabular}{ccc}
\hline Male & 24 & $68.6 \%$ \\
\hline Female & 11 & $31.4 \%$ \\
\hline Age median $=54$ & & \\
\hline$<54$ & 16 & $45.7 \%$ \\
\hline$>=54$ & 19 & $54.3 \%$ \\
\hline ECOG PS & & \\
\hline $0-1$ & 27 & $77.1 \%$ \\
$>=2$ & 8 & $22.9 \%$
\end{tabular}

Tumor location

\begin{tabular}{ccc} 
left & 27 & $77.1 \%$ \\
\hline right & 8 & $22.9 \%$
\end{tabular}

Histological differentiation

\begin{tabular}{ccc}
\hline Poorly & 2 & $5.7 \%$ \\
\hline Moderately & 31 & $88.6 \%$ \\
\hline Well & 2 & $5.7 \%$ \\
\hline Kras status & & \\
\hline Wild & 12 & $34.3 \%$ \\
\hline Mutant & 23 & $65.7 \%$ \\
\hline Number of metastatic organs & & \\
\hline$<=2$ & 18 & $51.4 \%$ \\
\hline$>2$ & 17 & $48.6 \%$ \\
\hline Liver metastasis & & \\
\hline Yes & 28 & $80.0 \%$ \\
\hline No & 7 & $20.0 \%$ \\
\hline Lung metastasis & & $42.9 \%$ \\
\hline Yes & 15 & \\
\hline
\end{tabular}


No $\quad 20 \quad 57.1 \%$

\section{Prior surgery}

\begin{tabular}{ccc} 
Yes & 24 & $68.6 \%$ \\
\hline No & 11 & $31.4 \%$
\end{tabular}

Prior bevacizumab

\begin{tabular}{ccc}
\hline Yes & 31 & $88.6 \%$ \\
\hline No & 4 & $11.4 \%$
\end{tabular}

Table 2 Response outcome

\begin{tabular}{ll} 
Outcome & $\mathrm{N}=35$ \\
\hline ORR (CR+PR),\% & $11.4 \%$ \\
\hline DCR (CR+PR+SD), \% & $60.0 \%$ \\
\hline DOR, median, mos & 3.4 \\
\hline PFS, median $(95 \% \mathrm{Cl})$, mos & $3.8(1.8-5.8)$ \\
\hline OS, median $(95 \% \mathrm{Cl})$, mos & $14.6(5.4-23.8)$
\end{tabular}

Table 3 Univariate analysis and multivariate analysis of clinical variable for the prediction of progression free survival 


\begin{tabular}{|c|c|c|c|c|c|}
\hline \multirow{2}{*}{$\begin{array}{l}\text { Variable } \\
\text { Total }\end{array}$} & & \multicolumn{2}{|c|}{ Univariate analysis } & \multicolumn{2}{|c|}{ Multivariate analysis } \\
\hline & & $H R$ & $\mathrm{p}$ & $\mathrm{HR}$ & $\mathrm{p}$ \\
\hline Gender & Male vs Female & $\begin{array}{l}0.924(0.435- \\
1.964)\end{array}$ & 0.837 & $\begin{array}{l}0.586(0.175- \\
1.956)\end{array}$ & 0.384 \\
\hline Age median $=54$ & $<54$ vs $>=54$ & $\begin{array}{l}0.712(0.351- \\
1.446)\end{array}$ & 0.348 & $\begin{array}{l}0.504(0.189- \\
1.341)\end{array}$ & 0.170 \\
\hline ECOG PS & $0-1$ vs $>=2$ & $\begin{array}{l}2.484(1.032- \\
5.978)\end{array}$ & 0.042 & $\begin{array}{l}2.760(0.819- \\
9.308)\end{array}$ & 0.102 \\
\hline Tumor location & Left vs Right & $\begin{array}{l}1.428(0.627 \text { - } \\
3.252)\end{array}$ & 0.396 & $\begin{array}{l}1.601(0.432- \\
5.930)\end{array}$ & 0.481 \\
\hline $\begin{array}{l}\text { Histological } \\
\text { differentiation }\end{array}$ & $\begin{array}{l}\text { Poorly vs Moderately } \\
\text { vs Well }\end{array}$ & $\begin{array}{l}1.780(0.437- \\
7.261)\end{array}$ & 0.421 & $\begin{array}{l}1.820(0.358- \\
9.250)\end{array}$ & 0.470 \\
\hline Kras status & Wild vs Mutant & $\begin{array}{l}1.172(0.556- \\
2.472)\end{array}$ & 0.676 & $\begin{array}{l}1.627(0.302- \\
8.769)\end{array}$ & 0.571 \\
\hline $\begin{array}{l}\text { Number of metastatic } \\
\text { organs }\end{array}$ & $<=2$ vs $>2$ & $\begin{array}{l}0.905(0.450- \\
1.817)\end{array}$ & 0.778 & $\begin{array}{l}0.920(0.353- \\
2.395)\end{array}$ & 0.864 \\
\hline Liver metastasis & Yes vs No & $\begin{array}{l}0.662(0.283- \\
1.548)\end{array}$ & 0.341 & $\begin{array}{l}0.592(0.182- \\
1.929)\end{array}$ & 0.384 \\
\hline Lung metastasis & Yes vs No & $\begin{array}{l}1.233(0.608- \\
2.498)\end{array}$ & 0.562 & $\begin{array}{l}1.655(0.565- \\
4.852)\end{array}$ & 0.358 \\
\hline Prior surgery & Yes vs No & $\begin{array}{l}1.441(0.671 \text { - } \\
3.091)\end{array}$ & 0.349 & $\begin{array}{l}1.906(0.466- \\
7.791)\end{array}$ & 0.369 \\
\hline Prior bevacizumab & Yes vs No & $\begin{array}{l}0.840(0.288- \\
2.447)\end{array}$ & 0.749 & $\begin{array}{l}1.547(0.359- \\
6.670)\end{array}$ & 0.558 \\
\hline
\end{tabular}

Table 4 Univariate analysis and multivariate analysis of clinical variable for the prediction of overall survival 


\begin{tabular}{|c|c|c|c|c|c|}
\hline \multirow{2}{*}{$\begin{array}{l}\text { Variable } \\
\text { Total }\end{array}$} & & \multicolumn{2}{|c|}{ Univariate analysis } & \multicolumn{2}{|c|}{ Multivariate analysis } \\
\hline & & $\mathrm{HR}$ & $\mathrm{p}$ & $\mathrm{HR}$ & $\mathrm{p}$ \\
\hline Gender & Male vs Female & $\begin{array}{l}0.660(0.253 \text { - } \\
1.720)\end{array}$ & 0.395 & $\begin{array}{l}0.603(0.118- \\
3.071)\end{array}$ & 0.542 \\
\hline Age median $=54$ & $<54$ vs $>=54$ & $\begin{array}{l}1.387(0.593- \\
3.242)\end{array}$ & 0.450 & $\begin{array}{l}1.242(0.401- \\
3.847)\end{array}$ & 0.707 \\
\hline ECOG PS & $0-1$ vs $>=2$ & $\begin{array}{l}0.639(0.214- \\
1.906)\end{array}$ & 0.421 & $\begin{array}{l}0.345(0.072- \\
1.664)\end{array}$ & 0.185 \\
\hline Tumor location & Left vs Right & $\begin{array}{l}0.881(0.324- \\
2.397)\end{array}$ & 0.804 & $\begin{array}{l}2.899(0.523- \\
16.065)\end{array}$ & 0.223 \\
\hline $\begin{array}{l}\text { Histological } \\
\text { differentiation }\end{array}$ & $\begin{array}{l}\text { Poorly vs Moderately } \\
\text { vs Well }\end{array}$ & $\begin{array}{l}0.866(0.265- \\
2.833)\end{array}$ & 0.811 & $\begin{array}{l}0.739(0.112- \\
4.860)\end{array}$ & 0.753 \\
\hline Kras status & Wild vs Mutant & $\begin{array}{l}0.439(0.165- \\
1.167)\end{array}$ & 0.099 & $\begin{array}{l}0.850(0.133- \\
5.439)\end{array}$ & 0.864 \\
\hline $\begin{array}{l}\text { Number of } \\
\text { metastatic organs }\end{array}$ & $<=2$ vs $>2$ & $\begin{array}{l}0.780(0.334- \\
1.822)\end{array}$ & 0.565 & $\begin{array}{l}0.673(0.213- \\
2.128)\end{array}$ & 0.500 \\
\hline Liver metastasis & Yes vs No & $\begin{array}{l}0.741(0.250- \\
2.197)\end{array}$ & 0.589 & $\begin{array}{l}0.552(0.115- \\
2.648)\end{array}$ & 0.458 \\
\hline Lung metastasis & Yes vs No & $\begin{array}{l}1.608(0.681 \text { - } \\
3.793)\end{array}$ & 0.278 & $\begin{array}{l}1.974(0.467- \\
8.348)\end{array}$ & 0.355 \\
\hline Prior surgery & Yes vs No & $\begin{array}{l}1.823(0.699- \\
4.750)\end{array}$ & 0.219 & $\begin{array}{l}1.984(0.437- \\
9.004)\end{array}$ & 0.375 \\
\hline Prior bevacizumab & Yes vs No & $\begin{array}{l}1.166(0.262 \text { - } \\
5.180)\end{array}$ & 0.840 & $\begin{array}{l}1.277(0.199- \\
8.192)\end{array}$ & 0.796 \\
\hline
\end{tabular}

\section{Figures}




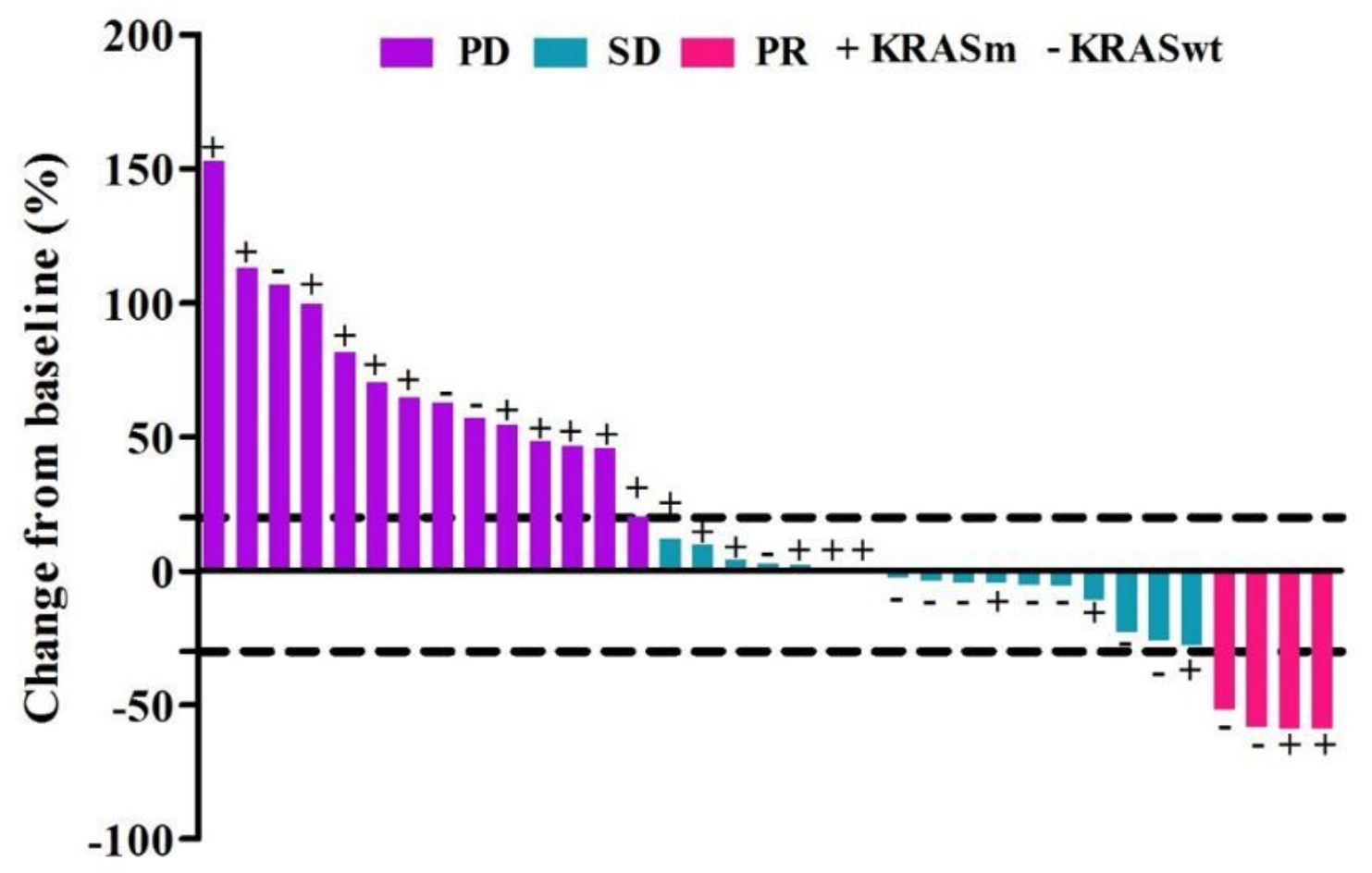

Figure 1

Tumor response in patients treated with fruquintinib plus pd-1 inhibitor with different kras status 
Strata + All

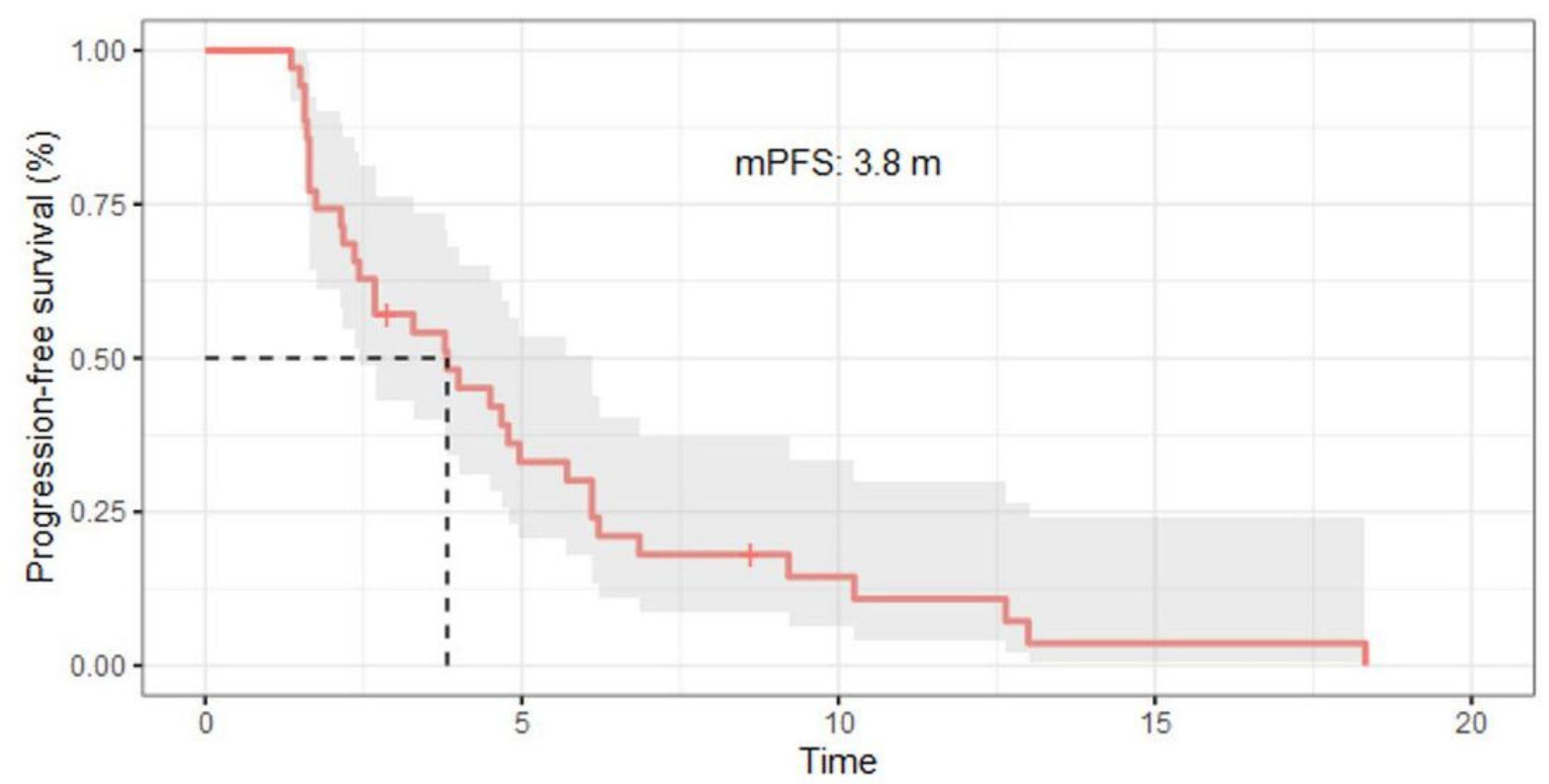

Number at risk

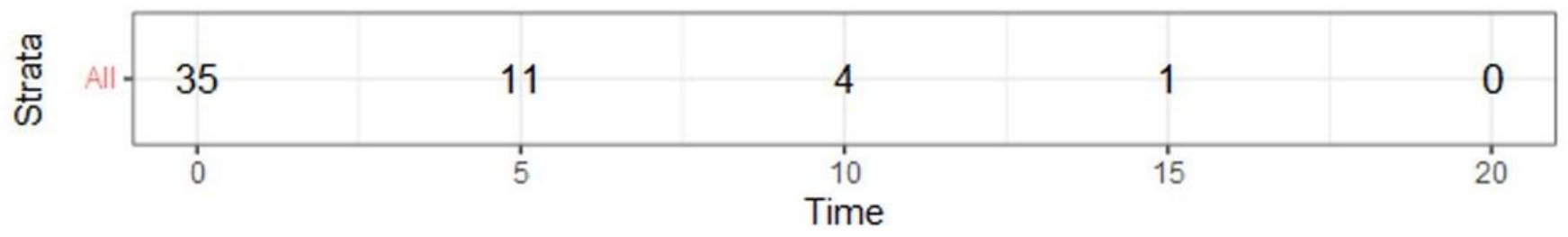

Figure 2

Progression free disease of patients treated with fruquintinib plus pd- 1 inhibitor 
Strata + All
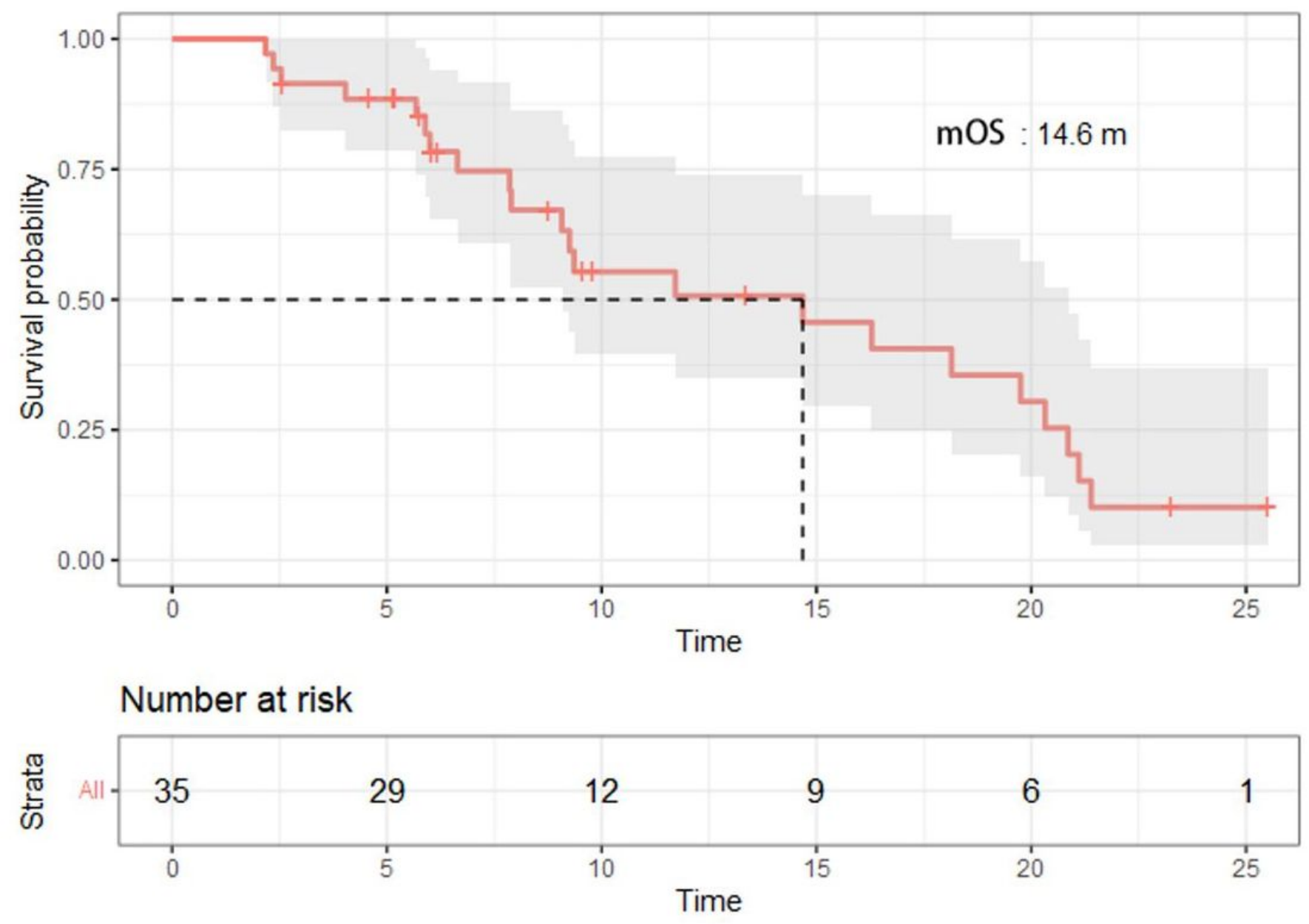

Figure 3

Overall survival of patients treated with fruquintinib plus pd-1 inhibitor 


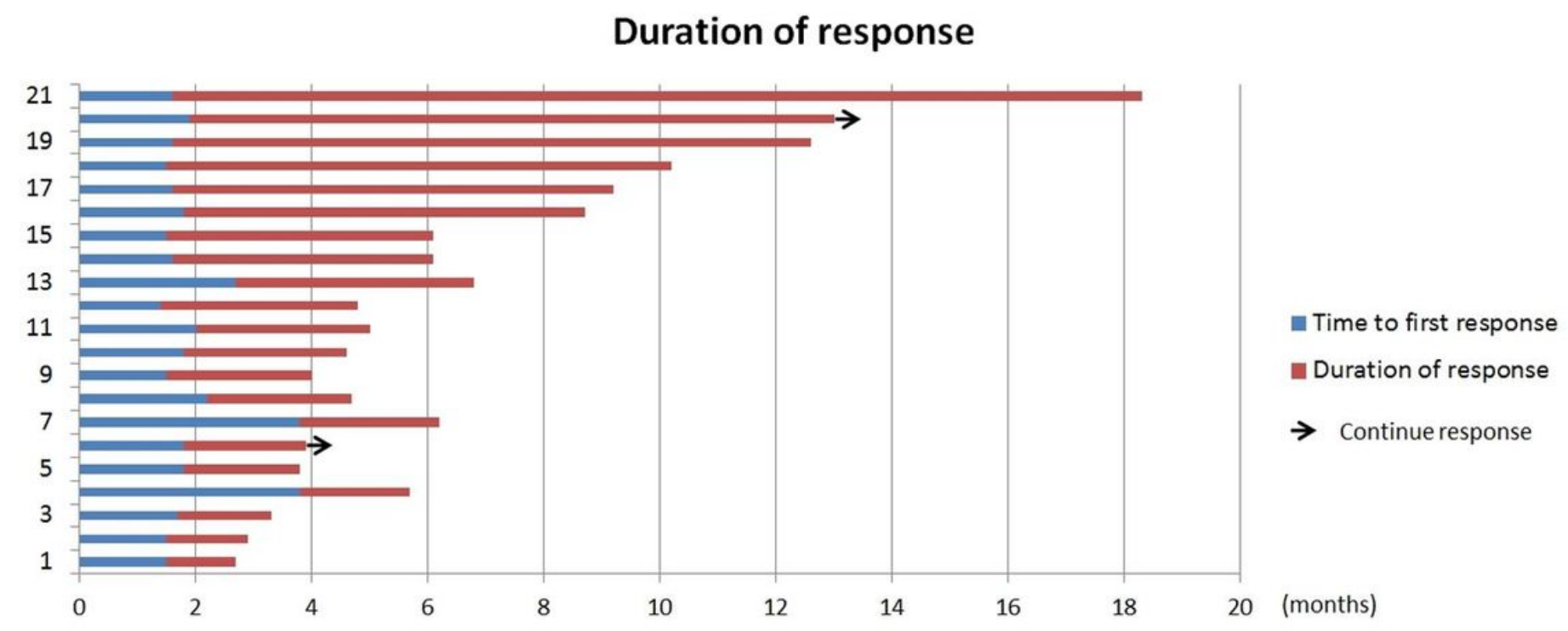

Figure 4

Duration of time of patients treated with fruquintinib plus pd-1 inhibitor 